\section{Effective Teaching Practices in Inclusive Classrooms}

\author{
Franziska Felder, Ariana Garrote, Helena Krähen- \\ mann, Elisabeth Moser Opitz, Susanne Schnepel, \\ Sarah Jandl, Universität Zürich
}

Gérard Bless, Universität Freiburg/CH

Rachel Sermier Dessemontet, HEP Vaud

Effective Teaching Practices in Inclusive Classrooms (intern Sirlus: Soutenir l'intégration - Integration unterstützen) ist eine vom Schweizerischen Nationalfonds SNF geförderte Längsschnittstudie in Integrationsklassen mit Kindern mit geistiger Behinderung. Untersucht werden zum einen die Effekte einer Intervention zur sozialen Integration und einer Leistungsintervention. Zum anderen interessiert der Zusammenhang zwischen Unterrichtsqualität, Zusammenarbeit der Lehrkräfte, Einstellung der Lehrperson zum Kind mit geistiger Behinderung und der Entwicklung der Lernenden.

\section{Forschungshintergrund}

Verschiedene gesetzliche Vorgaben und politische Entscheidungen (beispielsweise die Unterzeichnung und Ratifizierung der UN-Konvention über die Rechte von Menschen mit Behinderungen in Deutschland und Österreich, das Behindertengleichstellungsgesetz und das Sonderpädagogik-Konkordat in der Schweiz) führen zu verstärkten Integrationsbestrebungen. Zur Wirksamkeit von integrativer Schulung gibt es bereits wichtige Erkenntnisse. So gibt es Hinweise darauf, dass integrativ beschulte Kinder mit geistiger Behinderung dieselben oder stärkere Fortschritte im Leistungsbereich machen als separativ beschulte (Cole u.a. 2004; Freeman/Alkin 2000). Eine Studie aus der Schweiz (Sermier Dessemontet u.a. 2012) hat zudem gezeigt, dass Kinder mit geistiger Behinderung größere Fortschritte im sprachlichen Bereich machen, während im mathematischen Bereich keine Unterschiede zwischen den Settings nachgewiesen werden konnten. Allerdings zeigen empirische Forschungsergebnisse, dass schulleistungsschwache Schülerinnen und Schüler und Kinder mit geistiger Behinderung weniger gut sozial integriert sind, und zwar bezüglich Be- liebtheitsgrad, Freundschaften und gefühlter sozialer Integration (Pijl u.a. 2008; Huber 2006). Darüber hinaus kommen Unterrichtsstudien zum Schluss, dass die Einstellung der Lehrperson gegenüber Kindern mit geistiger Behinderung ein Schlüsselfaktor für deren erfolgreiche Integration darstellt (DeSimone/Parmar 2006; Huber 2011). Unterrichtsmerkmale wie Feedback oder Strukturierung des Unterrichts korrelieren zudem mit Interaktionen zwischen Lehrpersonen und Kindern mit geistiger Behinderung (Jordan u.a. 2010).

Trotz zahlreicher wichtiger Forschungsresultate bestehen aber auch Forschungslücken. So gibt es Forschungsbedarf hinsichtlich didaktischer Konzepte für den integrativen Unterricht, die nachweisbar die soziale Integration und die Leistungsentwicklung aller Kinder unterstützen. Gerade bei der integrativen Schulung von Kindern mit geistiger Behinderung besteht beispielsweise die Gefahr, dass sie in Schulstunden, in denen die Regellehrkraft allein ist mit der Klasse, nicht adäquat gefördert werden. Zudem zielen viele Vorschläge für die mathematische Förderung von Kindern mit geistiger Behinderung auf das Rechnen durch Abzählen ab (z.B. Calik/Kargin 2010) statt numerische Kompetenzen für die Alltagsbewältigung zu fördern. Auch ist wenig über die Unterrichtsqualität im integrativen Setting sowie über Interventionsmöglichkeiten bekannt. Forschungslücken betreffen weiter den Zusammenhang zwischen Einstellungen der Lehrpersonen bezüglich Behinderung, der Unterrichtsqualität, der Zusammenarbeit der Lehrpersonen und dem fachlichen Wissen (,subject matter knowledge“, „pedagogical content knowledge") der Sonderschullehrpersonen.

\section{Forschungsfragen}

Folgende Fragen werden im Projekt im zweiten Schuljahr untersucht:

1. Kann durch eine spezifische, von den Lehrkräften durchgeführte Intervention zur sozialen Integration (beispielsweise Feedbackverhalten, Thematisierung von Behinderung, spezifische Gruppenbildung) eine Verbesserung der sozialen Integration der Schülerinnen und Schüler erreicht werden? 
2. Kann durch eine spezifische, von den Lehrkräften durchgeführte Intervention im Mathematikunterricht (Materialien zur Differenzierung, Materialien, die für eine alltagsbezogene mathematische Förderung von Kindern mit einer geistigen Behinderung geeignet sind) Einfluss genommen werden auf die mathematische Entwicklung der Kinder mit geistiger Behinderung?

3. Über welches fachliche und fachdidaktische Wissen zum Mathematiklernen von Kindern mit geistiger Behinderung verfügen die Sonderschullehrpersonen?

4. Gibt es einen Zusammenhang zwischen der Einstellung der Regellehrperson zum Kind mit einer geistigen Behinderung, der Unterrichtsqualität, der Zusammenarbeit der Lehrpersonen und der Wirksamkeit der Integration?

\section{Forschungsdesign}

Es handelt sich um eine Interventionsstudie mit zwei Messzeitpunkten (Vortest Ende erstes Schuljahr und Anfang zweites Schuljahr; Nachtest Ende zweites Schuljahr).

Die Stichprobe wird aus ca. 60 zweiten Klassen aus der deutsch- und der französischsprachigen Schweiz bestehen, in denen jeweils mindestens ein Kind mit einer geistigen Behinderung $(I Q<75)$ integrativ unterrichtet wird. Die Klassen werden nach dem Zufallsprinzip den zwei Interventionsgruppen A (Mathematikintervention) und B (soziale Intervention) und einer Wartegruppe zugeteilt. Die Lehrpersonen der Interventionsgruppen nehmen an zwei Begleittreffen teil und setzen die Intervention ab Sommer 2014 bis im Frühjahr 2015 ein.

Erhoben werden folgende Daten:

Schülerinnen und Schüler ( $\mathrm{T}_{1}$ und $\left.\mathrm{T}_{2}\right)$ : Diverse personenbezogene Daten (Herkunft, Erstsprache usw.), Mathematikleistung, allgemeine Denkfähigkeiten (CFT1), soziale Integration (Nominationen, Rating, subjektives Wohlbefinden).

Regellehrpersonen (T1): Zusammenarbeit mit der Sonderschullehrperson, Einstellung zur Integration von Kindern mit einer Behinderung.
Sonderschullehrperson ( $\mathrm{T}_{1}$ ): Fachdidaktisches Wissen zur mathematischen Förderung von Kindern mit einer geistigen Behinderung.

In allen drei Gruppen wird im Verlauf der zweiten Klasse je eine Mathematiklektion videografiert.

Die ersten Erhebungen finden im Frühjahr 2014 statt. Die Studie dauert bis Sommer 2015.

Weitere Informationen sowie Literaturangaben können bei ffelder@ife.uzh.ch oder agarrote@ ife.uzh.ch eingeholt werden.

Informationen zum Projekt finden sich auch unter www.ife.uzh.ch/research/sbi/forschung/sirius info.html

\section{Möglichkeiten von Technik für Menschen mit Behinderung - phänomenologische Perspektiven}

\section{Robert Stöhr}

Universität zu Köln

\section{Technik - (k)ein Thema im Kontext Behinderung}

Unser Alltag wird bestimmt durch den Einsatz von zahlreichen Technologien. Allein das morgendliche Aufstehen, vom Wecker über das Bad zur Kaffeemaschine, involviert einen Apparat an ineinandergreifenden Systemen und Maschinen, der sich in seinem Funktionieren unserer Wahrnehmung weitgehend entzieht. In bestimmten Lebenssituationen, bspw. im Falle einer körperlichen Einschränkung oder einer Sinnesbehinderung, zeigen sich die Grenzen dieser technologischen Strukturen: das funktionale Zusammenspiel ist durchbrochen. Es werden Prothesen (in einem weiten Sinne verstanden als Hinzufügungen, Ersatzstücke oder Veränderungen am Körper oder auch in der Umwelt) notwendig, um buchstäblich „Anschluss“ zu gewährleisten.

Im Hinblick auf die Ermöglichung von Teilhabe, Selbstbestimmung und Unabhängigkeit von Menschen mit Behinderung nehmen kompensatorische Technologien eine hochgradig positiv besetz- 
te Bedeutung an. Die Umsetzung behindertenpolitischer Wandlungs- und Entwicklungsprozesse ist eng verzahnt mit der Bereitstellung und Weiterentwicklung dieser technischen Kompensationsmöglichkeiten. Als Assistive Technologien und als Maßnahmen zur Realisierung von Barrierefreiheit bzw. zu einer universell gestalteten Umwelt sollen sie ein Funktionieren und darauf basierende persönliche wie gesellschaftliche Lebensvollzüge ermöglichen helfen (vgl. Revermann/Gerlinger 2010).

Es zeigt sich eine Schwierigkeit: Wird, Technik' im Kontext von Technologien zur Verbesserung der Lebenssituation von Menschen mit Behinderung thematisiert, so artikuliert sich implizit oder explizit eine Grundannahme, die aus technikphilosophischer Sicht nur begrenzt gültig ist: ,Technik wird als bloßes Mittel verstanden. Diese Perspektive allein führt zu Fehleinschätzungen, die sowohl die Bedeutung von Technologien zur individuellen Rehabilitation und zur (Um)Gestaltung der Umwelt als auch die damit verknüpften behindertenpolitischen Zielvorstellungen und deren zugrunde liegendes Verständnis von Behinderung betreffen. Erste Spuren, die auf diese Problematik verweisen, zeigen sich in kulturwissenschaftlichen Überlegungen zur Bedeutung technischer Hilfsmittel sowie in Erfahrungsberichten von Menschen mit Behinderung (vgl. z. B. Bösl 2012, 5of; Maskos 2011, 8). Die Konturen des insbesondere im politischen Diskurs selbstverständlich positiv besetzten Technikbegriffs werden unscharf.

Vor diesem Hintergrund problematisiert die Untersuchung das Fehlen einer fundierten theoretischen Auseinandersetzung mit dem Begriff ,Technik' im Kontext Behinderung. Ohne selbige wird weder die Einseitigkeit dieses Technikverständnisses und dessen begrenzte Reichweite sichtbar, noch kann eine angemessene Reflexion der Möglichkeiten und Bedeutungen von ,Technik'stattfinden.

\section{Erläuterung, Entwicklung und Klärung der Problemstellung}

Ein erster Schritt versteht sich als Skizze verschiedener Dimensionen dessen, was unter ,Technik' verstanden werden kann: Bis heute versteht sich der Begriff, Technik' noch oft als
Abstraktionsbegriff für das „Arsenal von Mitteln" (Waldenfels 1990, 149), das Techniker uns zur Verfügung stellen. Gegenwärtige technikphilosophische und -soziologische Überlegungen deuten ,Technik' hingegen als Begriff für den umfassenden Vermittlungszusammenhang zwischen Menschen und Dingen: Es geht nicht nur um eine Sammlung von Maschinen, Verfahrensweisen und Werkzeugen, sondern eher um die Art und Weise, wie sich das Zusammenspiel von Mensch und Welt ausgestaltet. Daher muss der Technikbegriff in einen handlungsbezogenen Kontext gestellt werden, der zeigt, dass „Technik ein anderes Wort dafür [ist], dass Menschen und Dinge zusammenarbeiten - so wie Sprache ein anderes Wort dafür ist, dass Menschen mit anderen Menschen kommunizieren" (Nordmann 2008, 70). Ein ausschließlich instrumentelles und auf „Technikdinge“ begrenztes Technikverständnis sowie die daraus resultierende Fokussierung bloßer Mittel-Zweck-Schemata erweisen sich mit Blick auf die Einschätzungen der Möglichkeiten konkreter Technologien als ungenügend.

Blickt man vor diesem Hintergrund in einem zweiten Schritt auf die Thematisierung von ,Technik' im Kontext Behinderung, so zeigt sich:

- In der Grundlagenliteratur fehlt eine Auseinandersetzung mit dem Begriff ,Technik' weitgehend.

IIm Zusammenhang mit der Anwendung technischer Hilfen wird ,Technik' als bloßes Instrument zur Umsetzung vorbestimmter (z.B. behindertenpolitischer) Ziele verstanden.

- Kulturwissenschaftliche Perspektiven zeigen, dass eingedenk kulturhistorischer Kontexte technologische Entwicklungen ambivalente Wirkungen zeitigen können.

- Berichte von Menschen mit Behinderung verweisen auf paradoxe Erfahrungen mit technischen Dingen.

Der Dominanz einer instrumentellen Deutung stehen einige wenige Ansätze entgegen, die diese Deutung strittig werden lassen. Eine angemessene Reflexion dessen, was jeweils mit dem Begriff ,Technik' oder ,technisch“ verbunden wird, fehlt dennoch. Das Fehlen dieser Reflexion selbst muss als Ausdruck einer unter- 
stellten Selbstverständlichkeit des Technikbegriffs gedeutet und hinterfragt werden (vgl. Waldenfels 1990, 137). Die bestimmten Technologien unterstellten Möglichkeiten hinsichtlich Rehabilitation und Teilhabe, Selbstbestimmung und Zugänglichkeit werden fragwürdig, wenn keine Verständigung darüber stattfindet, was ,Technik' bedeutet.

Im dritten Schritt werden zunächst Ursachen dieses Fehlens ausgeleuchtet: Die schon in der Antike wurzelnde Idee vom Menschen als erkennendes Subjekt und von der Welt als erkennbares Objekt führen in der Reflexion zu einem verzerrten Gegenstandsbezug (vgl. Ihde 1979, 3). „Mit den Dingen handeln“ bedeutet dann nur mehr eine zeitweilige Synthese von Mensch und Ding, wobei einmal der Mensch, das andere Mal das Ding die Oberhand behält. Aus dieser einen Quelle, diesem dichotomen Verhältnis, schöpfen noch heute Technikskeptiker bzw. Technikoptimisten ihre Argumente.

Eine phänomenologische Analyse des Zusammenwirkens von Menschen und Dingen zeigt die Genese einseitiger Technikbegriffe auf. Als verleiblichte Subjekte sind Menschen immer schon in der Welt verankert und mit den Dingen verflochten (vgl. Merleau-Ponty 1965, 367ff). In diesem Sinne arbeiten Menschen je schon mit den Dingen, mit der Welt zusammen, sodass jegliche Wahrnehmung und Handlung in einem weiten Sinne als technisiert verstanden werden kann (vgl. Waldenfels 1990, 144).

Diese phänomenologischen Überlegungen eröffnen eine erweiterte Perspektive zur Reflexion auf die Möglichkeiten von Technologien. Darüber hinaus lassen sie sichtbar werden, inwiefern ,Technik' an der Herausbildung der sozialen Differenzierungskategorie Behinderung beteiligt ist (vgl. Bösl 2012, 50). Wird die Technizität menschlichen Wahrnehmens, Denkens und Handelns berücksichtigt, so wird deutlich, dass ,Technik' in unserem Verhältnis zu uns selbst und zu unserer Welt von Beginn an ihre Spuren hinterlässt und dabei „Spielräume“ eröffnet, die weder als nur begrenzt noch als nur grenzenlos zu verstehen sind.

\section{Zielstellung}

In der vorliegenden Arbeit soll gezeigt werden, dass die theoretische Auseinandersetzung mit dem Begriff ,Technik' im Kontext Behinderung unabdingbar ist, weil nur so die weitreichende Bedeutung von ,Technik' für das, was wir unter Behinderung verstehen, herausgearbeitet und bei der Reflexion auf die Möglichkeiten des Einsatzes von Technologien berücksichtigt werden kann. Die Arbeit problematisiert das Fehlen einer notwendigen systematischen Auseinandersetzung mit dem Begriff, Technik' und thematisiert dessen Bedeutung in Wissenschaften, die sich mit Behinderung auseinandersetzen. Vor dem Hintergrund technikphilosophischer und phänomenologischer Überlegungen soll mithilfe einer Literaturanalyse besagtes Fehlen herausgestellt und problematisiert werden, ehe dann eine Neuausrichtung/Erweiterung der Perspektive vorgeschlagen wird. So soll eine einseitige und in der Folge verzerrte Reflexion auf die Möglichkeiten technischer Hilfen vermieden und ein Nachdenken über ,Technik' möglich werden, das deren Bedeutung für soziale Prozesse allgemein, aber besonders in Hinblick auf Menschen mit Behinderung in ein anderes Licht rückt.

Weitere Informationen und Literaturhinweise können eingeholt werden bei rstoehr@unikoeln.de 